\title{
La producción mediática como método constructivo de lo social, apuntes para una Comunicología integrativa
}

\author{
José Luis Martin Galindo* \\ Universidad del Claustro de Sor Juana
}

\section{RESUMEN}

Se considera que al articular la historia de los medios ciudadanos y la comunicación alternativa en América Latina con la Comunicología contemporánea, emergen elementos para el desarrollo de un modo de investigación, en el que se recurra a la producción audiovisual como un cúmulo de saberes pertinentes para proyectos estratégicos de reestructuración de tejidos sociales. Se propone una Comunicología integrativa, generada desde una metodología en la que se articulan dos perfiles de comunicólogo tradicionalmente escindidos en nuestro campo, por un lado el técnico o artista productor de contenidos y por el otro el investigador de la comunicación social.

Palabras clave: medios ciudadanos, Comunicología, investigación integrativa, transformación social

\begin{abstract}
It is considered that by articulating the history of citizen media and Latin American alternative communication with contemporary Communicology, elements emerge to develop an inquiry mode in wich one can recurse to audiovisual production as a relevant body of kwowledge for social change projects. An integrative Communicology is proposed, in wich two traditionally separated professional profiles are combined: on the one hand the technical or artistic content producer, on the other, the social communication researcher.
\end{abstract}

Keywords: citizen media, Communicology, integrative research, social transformation

*Licenciado en Comunicación por la Universidad Autónoma de Baja California; cursó el Diplomado Internacional en Documental de Creación Mirada de Dos Mundos, de la Universidad del Valle, en Cali, Colombia; es maestrando en Pensamiento Complejo de la Multiversidad Mundo Real Edgar Morin. Correo electrónico: jlmartin@elclaustro.edu.mx 


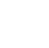

\section{PUNTO DE PARTIDA}

Aquí y ahora, ante el constante reordenamiento del mundo, se experimenta el campo de la Comunicación entre una revolución informática que nunca se alcanza por completo y la emergencia de nuevos paradigmas en el pensamiento científico, desde un contexto regional mexicano, latinoamericano, marcado por fuertes crisis económicas, políticas, socioculturales, que resultan imposibles de ignorar pues trastocan los diversos ámbitos de nuestra vida cotidiana.

Desde tal escenario se piensa en los puntos de encuentro histórico entre la producción audiovisual (y mediática en general) y la generación de conocimiento en las Ciencias Sociales, lanzando la pregunta por el tipo de investigación que se puede generar desde el campo actual de la Comunicación, para aportar comprensión y construir conocimiento acerca de los procesos de intercambio sociocultural como aspecto central de nuestras complejas realidades, y que además provea herramientas para algunos procesos de transformación social, dirigidos hacia el empoderamiento comunicacional de comunidades, colectivos u organizaciones.

Lo anterior implica retomar la relación entre el audiovisual y las Ciencias Sociales, que es tan antigua como el mismo cinematógrafo

\section{José Luis Martin Galindo}

de Lumière (creado en 1895), el cual posibilitaría la práctica de recoger en imágenes la cultura material (Pinto, 1999, p. 80) y al mismo tiempo incidir en los fenómenos inmateriales de lo social. En este sentido, Félix-Louis Régnault ya hablaba en 1931(con un optimismo un tanto desbordado) del potencial de los dispositivos de grabación y filmación como herramientas de investigación:

Films y fonogramas son materiales que utilizarán, en un futuro muy cercano, todos los sabios de las ciencias humanas. Gracias a ellos, el psicólogo, etnólogo, sociólogo, lingüista, folklorista coleccionan en sus laboratorios todos los comportamientos de numerosas etnias y pueden evocar a su gusto la vida de éstas. Analizando, mensurando estos documentos objetivos, comparándolos, seriándolos, llegando a establecer los métodos convenientes para sus ciencia, para conocer las leyes de la mentalidad humana (cit. en Pinto, 1999, p. 81).

Si bien es cierto que tal predicción no fue del todo certera, se coincide con la inquietud de seguir explorando las potencialidades de la producción mediática como herramienta de indagación, al considerar que entre la historia de la comunicación alternativa y los medios ciudadanos de América Latina, desarrollada 
La producción mediática

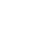

durante la segunda mitad del siglo XX, así como en la Comunicología contemporánea, se encuentran elementos para articular un estilo de investigación que recurra a la producción audiovisual como un cúmulo de saberes pertinentes para proyectos estratégicos de observación, descripción y reestructuración de tejidos sociales.

Se propone entonces una Comunicología integrativa en la que se articulan dos perfiles de comunicólogo tradicionalmente escindidos en nuestro campo: por un lado el técnico o artista realizador de contenidos y por el otro el investigador de la comunicación social. El objetivo es plantear una modalidad de ejercicio profesional que nos permita combinar ambos oficios para fungir como gestores y operar desde el acompañamiento de proyectos para los que las estrategias de intervención comunicológicas puedan ser relevantes.

\section{PRODUCCIÓN DESDE MEDIOS CIU- DADANOS}

En la historia de la Comunicología latinoamericana, la producción independiente influída por el pensamiento crítico tanto en la radio, el cine, el video y en general los medios no hegemónicos de información y difusión, ha jugado un rol importante en los procesos de democratización, de libre participación, de mo-

\section{José Luis Martin Galindo}

dos horizontales y colaborativos de producción, que se han implementado históricamente en respuesta a los modelos de desarrollo, a los sistemas dictatoriales instaurados en la región durante el siglo pasado, o a las violentas democracias en las que vivimos muchos habitantes de esta región.

Son muchos los términos que se han utilizado para nombrar esos proyectos mediáticos comprometidos con los movimientos sociales, sea comunicación alternativa, ciudadana, comunitaria, participativa, popular, entre otros. Clemencia Rodríguez ha planteado una diferenciación entre medios ciudadanos, comunitarios y alternativos que resulta útil:

El término "medios ciudadanos" define los procesos mediáticos a partir de su potencial para desencadenar procesos de cambio social. Por el contrario, el concepto de "medios comunitarios" los define a partir de quiénes los producen (es decir, organizaciones comunitarias, colectivos de base, etc.) o del tipo de licencia de difusión que el estado les otorga (por ejemplo, licencia de transmisión comunitaria). A su vez, el término "medios alternativos" define los medios en tanto lo que no son (por ejemplo, alternativos frente a los medios dominantes, alternativos frente a la 


\section{-}

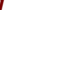

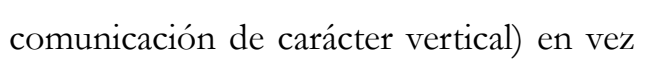

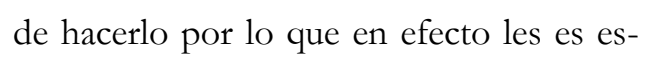
pecífico (Rodríguez, 2009, pp. 17-18).

De acuerdo con la anterior concepción de medios ciudadanos, se les puede considerar como herramientas para incidir en las relaciones entre medios, política y ciudadanía, pues abarca:

Todos aquellos medios que abren espacios de comunicación donde hombres, mujeres, niñas y niños aprenden a manipular sus propios lenguajes, códigos, signos y símbolos, y adquieren poder para nombrar el mundo en sus propios términos. Los medios ciudadanos activan procesos a través de los cuales individuos y comunidades re-codifican tanto sus contextos como su propia identidad (Rodríguez, 2009, p. 19).

En cuanto al concepto de ciudadanía, éste se ha transformado en las últimas décadas, de tal forma que actualmente se concibe más allá de las normativas y los derechos reconocidos por el aparato estatal.

En los años 90 cuando vamos a empezar a hablar de esas nuevas ciudadanías, que de
José Luis Martin Galindo alguna manera revisaron su relación con el Estado; (...) que desde entonces adquieren mucha más autonomía, mucha más fluidez, mucha más independencia; (...) que hoy las encontramos en distintos escenarios: las ciudadanías sexuales, étnicas, medioambientales, (...) y las ciudadanías mediáticas (Téllez, 2013, 16:09-17:42).

La noción de ciudadanías mediáticas pone sobre la mesa algunos cuestionamientos relacionados con la presencia de los ciudadanos en los medios masivos, con las formas en que son representados, con la visibilidad o invisibilidad de éstos, tanto en la radio, la televisión, la prensa, el cine y por supuesto la internet. Y aquí cabe evocar la noción de nueva ciudadanía que propone la brasileña Evelina Dagnino:

La nueva ciudadanía (...) no está vinculada a una estrategia de las clases dominantes y del Estado de incorporación política gradual de los sectores excluidos, con el objetivo de una mayor integración social o como una condición legal y política necesaria para la instalación del capitalismo. La nueva ciudadanía requiere -inclusive es pensada en ese proceso- la constitución de sujetos sociales activos (agentes políti- 


\section{La producción mediática}

.

cos), que definen lo que consideran son sus derechos y luchan por su reconocimiento en cuanto tales. En ese sentido, es una estrategia de los no ciudadanos, de los excluidos, una ciudadanía "desde abajo" (2004, p. 15).

Las nociones que manejan Téllez y Dagnino ayudan a preguntarse por todas aquellas formas de ciudadanía que se articulan hoy día como estrategias de transformación social, de resistencia, o incluso supervivencia ante los efectos más adversos del sistema político y económico también en México.

Podemos pensar entonces en la posible noción general de ciudadanías resilientes y en las posibles estrategias mediáticas correspondientes al ejercicio de dichas ciudadanías, naturalmente vinculadas a la defensa del derecho de libertad de opinión y expresión, al derecho a la información y al derecho a la comunicación.

Las estrategias mediáticas referidas se vinculan con las prácticas que hoy englobamos bajo la noción de videoactivismo, en el contexto de una revolución informática en pleno desarrollo y acelerada expansión. Concepción Mateos y Carmen Gaona distinguen tres modos básicos de este tipo de prácticas sociales de carácter comunicativo:

\section{José Luis Martin Galindo}

\begin{abstract}
Mientras el videoactivismo es una práctica audiovisual de intervención política sobre realidades no sólo relativas a los medios, el activismo mediático es una actividad de lucha por la transformación del sistema de medios con criterios de democratización y el activismo de medios autónomos se refiere a la producción de medios ajenos al sistema mediático hegemónico o dominante para comunicar al margen de él (Uzelman, cit. en Mateos y Gaona, 2015, p. 132).
\end{abstract}

La historia de los medios ciudadanos en América Latina está profundamente vinculada a una praxis comunicológica que ha ofrecido otras lecturas de nuestras realidades socioculturales, caracterizadas por una serie de reconstrucciones históricas hechas desde las particularidades idiosincráticas de cada país, así como por la relativización y toma de distancia ante las teorías anglosajonas o europeas.

De esta historia podemos aprender lo que la gente, las comunidades, los ciudadanos han venido haciendo con los medios de producción de contenidos y de difusión a su alcance para transformar sus realidades compartidas, desde la participación en procesos pedagógicos, de sistematización de experiencias, de trabajo con grupos políticos de base, o proyectos de investigación-acción. Esto con el fin de se- 


\section{La producción mediática}

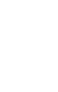

\begin{abstract}
guir pensando y desarrollando el audiovisual, no solo como herramienta de observación-autoobservación y de narración, sino como elemento catalizador de la interacción, desde adentro de comunidades de interés y expresiones heteronómicas de diversa índole.
\end{abstract}

\section{COMUNICOLOGÍA DESDE UN EN- FOQUE CONSTRUCTIVO DE LO SO- CIAL}

En el campo de la Comunicación, autores como Antonio Pasquali, Paulo Freire, Armand Mattelart, Luis Ramiro Beltrán, Rosa María Alfaro, Marita Matta, Néstor García Canclini, Mario Kaplún, y Jesús Martín Barbero (por mencionar algunos) desarrollaron, a partir de los años 60 , algunos conceptos o teorías que le han permitido a Latinoamérica pensar la cultura y los medios de comunicación de masas en sus propios términos (Rodríguez, 2009, p. 14).

Las propuestas de estos autores y autoras derivaron en una serie de estudios comprometidos con los movimientos sociales, con los que se ha buscado promover la construcción de sociedades más justas e igualitarias, intentando reducir, con mayor o menor éxito, las relaciones de dependencia y subalternidad de los grupos menos favorecidos.

Luis Ramiro Beltrán propuso un modelo de comunicación horizontal, que se aleja de una concepción difusionista de los procesos de comunicación para entenderlos desde un enfoque más complejo, con el que reivindica su cualidad humana, así como la condición democrática inherente a los mismos (Torrico, 2014, p. 5), por lo que se vuelve un referente obligado para la presente propuesta.

La llamada Escuela Crítica Latinoamericana, de la que Luis Ramiro Beltrán fue pionero, cuestionó la comunicación dominante, el sistema vertical de imposición de imaginarios, como uno de los principales problemas para el progreso en la región y defendió la comunicación como ejercicio de liberación y gobernanza ciudadana (Barranquero, 2014, p. 10).

En 1997 un grupo interdisciplinario que incluía al comunicólogo Alfonso Gumucio planteó la noción de comunicación para el cambio social para referirse a una forma de comunicación inclusiva que permite a la gente apropiarse de los proyectos mediáticos. Lo esencial de este tipo de comunicación, arguye Gumucio, "es que cuestiona el concepto de un desarrollo que no cuente con la participación de los sectores directamente afectados, y promueve una comunicación que haga efectiva la participación comunitaria, particular- 


\section{La producción mediática}

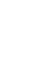

mente de los sectores más pobres y aislados" (2011, p. 28).

Este tipo de comunicación otorga mayor relevancia al proceso de producción que a los productos resultantes, una vez que los actores sociales se apropian del rol de comunicadores, por lo que su participación en la toma de decisiones editoriales y realización de contenidos se considera un elemento más de crecimiento colectivo.

Desde la comunicación para el cambio social se contempló la necesidad de un "nuevo comunicador para el siglo XXI" que se caracterizaría, entre otros aspectos, por mantener el equilibrio entre teoría y práctica, conocer las técnicas de comunicación, desarrollar un pensamiento estratégico de largo plazo, comprender a la tecnología como una herramienta más, asumir un rol de servicio como facilitador de procesos, más que un rol de creador (Gumucio, 2011b, 08:50-09:30).

Pero estudiar la comunicación no sólo es pensar lo mediático, pues ésta "puede concebirse como la interacción mediante la que gran parte de los seres vivos acoplan sus conductas frente al entorno, se adaptan a él" (Rizo, 2007, p. 13). Así, se retoma aquí la figura de un comunicólogo que va más allá del ejercicio sólo como analista de los medios masivos o como productor de mensajes y contenidos, concep- ciones del comunicador construidas a lo largo de la historia de las Ciencias de la Comunicación desde:

Dos campos de enseñanza casi deslindados por completo: el desarrollo de habilidades técnicas para el manejo de los medios de comunicación y la enseñanza de teorías de la comunicación combinadas con fundamentos sociológicos, filosóficos, crítica ideológica y todo aquello que suene a cultura general (Macías y Cardona, 2006, párr. 7).

Es decir que, por un lado, está el perfil operativo-técnico necesario para toda empresa o proyecto de medios masivos, y por otro aquel más cercano a la figura de un crítico o científico social.

Ya entrado el presente siglo, Luis Jesús Galindo Cáceres (2009, 2011, 2014, 2015) junto con una colectividad de investigadores conformó el programa Hacia una Comunicología Posible (HCP), el Grupo Hacia una Comunicología Posible (GUCOM) y el Grupo Hacia una Ingeniería en Comunicación Social (GICOM), buscando delimitar una ciencia general de la comunicación, así como su complemento ingenieril, precisamente la Ingeniería en Comunicación Social, con el fin de "desarrollar una metodología que permita promover el campo de la acción social hacia 


\section{-}

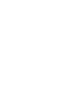

niveles de operatividad y eficiencia mayores que los que tenemos hoy día" (Galindo, 2015b, pár. 2).

Norma Macías y Diana Cardona son dos de las investigadoras cercanas a Galindo, quienes también han subrayado la importancia de que el estudio de la comunicación se haga en términos científicos, y que la práctica de ésta cuente con un fundamento conceptual, metodológico y técnico con sustento en la Comunicología.

Para nosotros la Comunicología en desarrollo, su estudio y su práctica, no tiene sentido si no la entendemos como ciencia aplicada que depende de otras, pero que aporta su propia visión y su forma de implementarse en la sociedad, como lo hace la ingeniería o la medicina, y que, al igual que éstas, deberá enfocarse al diagnóstico y resolución de problemas sociales (Macías y Cardona, 2011, párr. 25).

Jesús Galindo complementa:

La intención general en principio consiste en operar sobre espacio-tiempo sociales en las figuras generales de la intervención y el acompañamiento activo, con todos los ajustes que sean necesarios y pertinentes para hacerlo. Si hay algún problema en el orden de lo que entendemos co-

\section{José Luis Martin Galindo}

mo social la Ingeniería Social debe ser capaz de proponer alguna solución, y no sólo una, sino un paquete de posibilidades dentro de un gradiente del diseño general de soluciones posibles, con un diverso y variado paquete de aplicaciones técnicas para los diversos escenarios de futuros pertinentes y/o deseables. Problemas y soluciones, exploración problemática y diseño de soluciones. En el caso particular de la Ingeniería en Comunicación Social con la perspectiva del sentido de la comunicación como guía, es decir, la Comunicología (Galindo, 2014, p. VII).

Coincidimos con Macías, Cardona y Galindo al considerar nuestra labor profesional desde una perspectiva tan cientista como interesada en el mejoramiento o la transformación de nuestras posibles formas de ser y estar en sociedad. Para poner en práctica los procesos de intervención social necesarios en toda aplicación comunicacional de la Ingeniería Social, los autores mencionados desarrollaron a principios de siglo la noción de Comunicometodología.

Todo lo que aprendemos de las formas de composición y organización de mundo nos permite imaginarnos mundos alternativos, distintos, semejantes, que puedan llegar a ser bajo ciertas condiciones, con cierto gasto de energía y con la 


\section{La producción mediática}

.

.

aplicación de ciertos principios constructivos eficientes. La comunicometodología es esa perspectiva tecnológica de construcción de lo posible. Por una parte, aprendemos cómo es que ponemos en común lo que nos configura como entidades socioculturales (la comunicología), y por otra, a partir de ese aprendizaje podemos intervenir las formas de esa construcción y configurar otras, alterar las que tenemos, confirmar y reforzar nuestros procesos de configuración (la comunicometodología) (Galindo, 2005, pp. 15-16).

A partir de una revisión, de un diálogo a la distancia con las propuestas del GICOM se puede pensar en la posibilidad de un enfoque constructivo de lo social, conformado por una serie de soluciones técnicas para problematicas vinculadas a necesidades de desarrollo comunicacional, en las que se pueda recurrir a la producción mediática, a la realización audiovisual gestionada desde el acompañamiento de colectividades, como recurso que aporte elementos para ajustar o desajustar diversas formas de interacción, situadas en contextos específicos, practicadas por sistemas sociales delimitables, e históricamente construidas.

Es en dicho sentido que nos ha parecido pertinente revisar tanto las propuestas de Galindo y colegas, como los conceptos y metodologías heredadas de los medios ciudadanos
José Luis Martin Galindo

en América Latina, al proveernos esta última con múltiples casos posibles de estudio, de los que se puede aprender cómo ha sido aplicada, o cómo podría aplicarse la producción mediática en dichos ajustes de las formas de interacción, desde un enfoque constructivista que puede vincularse a una posible Ingeniería en Comunicación Social.

\section{HACIA UNA COMUNICOLOGÍA IN- TEGRATIVA}

A partir de la noción de Comunicología integrativa se propone la delimitación de un modo de investigación en el que se recuperan algunas prácticas heredadas de las culturas mediáticas colaborativas y comprometidas con el mejoramiento de las circunstancias sociales, tan presentes en la historia latinoamericana, y que se han materializado en los medios alternativos, comunitarios, ciudadanos, en la comunicación para el cambio social y en el videoactivismo, entre otras formas de expresión, difusión e intervención. Esto, como ya se ha establecido, desde un acercamiento a lo que viene proponiendo el GICOM desde su labor campal, que en palabras del mismo Galindo: "se trata de que la visión sobre la comunicación construya y reconozca guías e instrumentos prácticos de acción, intervención y acompañamiento en la vida social" (2017, p. 4). 


\section{-}

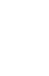

Es así que en el modo o estilo de investigación que se propone la producción audiovisual es concebida como espacio de intercambio, como recurso estratégico, como dispositivo comunicológico y como herramienta comunicometodológica que nos permita implementar "visiones prácticas, operativas, eficientes, para armar nuestros aparatos e instrumentos para resolver los problemas de la vida social" (Galindo, 2011, p. 205), en la que se articulan:

- El comunicólogo como científico social

- El comunicólogo como productor de medios

A partir de vincular:

- La producción mediática,

- La Comunicología aplicada en intervenciones-acompañamientos sociales.

Cuando se habla del audiovisual como posible herramienta comunicológica se hace referencia a un producto mediático que puede ser de factura doméstica y por lo tanto de (relativamente) bajo presupuesto, que va más allá de los medios ciudadanos entendidos como formas o textos audiovisuales que suelen dedicarse exclusivamente a la denuncia de injusticias, y más acá del audiovisual etnográfico, entendiendo a éste como un texto susceptible de convertirse en objeto de estudio o en herramienta para una investigación de corte antropológico o sociológico, en las que generalmente se busca representar o analizar los comportamientos de grupos culturales en observación.

La Comunicología integrativa avanzaría en otro sentido complementario, al concebir a la realización audiovisual como parte de una estrategia comunicacional para acompañar la autobiografía colectiva de un grupo de personas que deciden emprender un proyecto transformador. Es decir que desde la figura de un comunicólogo-gestor se promueve el aprovechamiento de los recursos informacionales, mediáticos y comunicacionales presentes en los contextos cotidianos de la colectividad con la que se pretende trabajar.

El comunicólogo-gestor funge también como (o incluye en su equipo a) un realizador que lo auxilia a investigar creando y produciendo objetos culturales audiovisuales, mediante un proceso colaborativo en el que la comunidad se puede integrar como parte del crew o equipo de producción para hacer video, televisión, o cine por sí mismo, apropiándose de las herramientas tecnológicas a la mano, así como de las técnicas, los formatos y lenguajes cinematográficos o televisivos socializados en el proceso de intervención-acompañamiento. 
Desde la Comunicología integrativa el comunicólogo aborda la tarea de gestionar procesos de alfabetización mediática y/o de apropiación tecno-mediática, es decir:

\footnotetext{
Prácticas a través de las cuales los sujetos (individual y colectivamente, desde las organizaciones sociales, políticas y sindicales), habiendo realizado una elucidación crítica acerca de los condicionamientos económicos, tecnológicos, sociales e ideológicos que acompañan la presencia de los medios de comunicación y las TIC existentes en su contexto inmediato y los discursos que ellos vehiculizan, expresan en la creación y uso de nuevos medios y discursos, su deseo y libertad de manifestar sus propias necesidades, convicciones e intereses, en el marco de la construcción de proyectos de autonomía individual y colectiva (Morales, 2009, p. 1).
}

Regresando a Galindo (2005), este autor establece una clasificación de cuatro dimensiones comunicológicas en las que se pueden organizar idealmente las prácticas de comunicación, y alrededor de las que giran la indagación y la producción de la Comunicología como ciencia aplicada:

- Expresión. Tiene que ver con la pro- ducción de cualquier tipo de mensaje y sus aspectos formales.

- Difusión. Tiene que ver con lo que se hace con medios de información, y lo que la gente hace con lo que se hace desde dichos medios.

- Interacción. Tiene que ver con la afectación, a partir de interrelaciones mutuas, entre individuos o sistemas sociales diversos.

- Estructuración. En esta dimensión se interrelacionan las anteriores para lograr una comprensión más compleja de los sistemas sociales y construir conocimientos útiles, es decir una Comunicología, para aplicaciones posteriores desde una Comunicometodología, necesarias para una Ingeniería en Comunicación Social.

La metodología que proponemos ubica a su vez cuatro tipos de acción general en la que se recurre a los medios audiovisuales como herramienta para el desarrollo o empoderamiento comunicacional de la colectividad en la que se trabaje:

- Observación o autoobservación del sistema social o comunidad (corresponde a la dimensión de la Interacción). 
La producción mediática

\begin{abstract}
presentación
\end{abstract}
- Representación o autorepresentación (dimensión de la Expresión).

- Sistematización de experiencias de intervención-acompañamiento (dimensión de la Difusión).

- Articulación o desarticulación de sistemas sociales (dimensión de la Estructuración).

Por ejemplo, en el caso de que el problema comunicacional a resolver o intervenir en la comunidad de interés con la que se trabaje pertenezca a la dimensión comunicacional de la expresión, es decir, en el caso de que la colectividad en cuestión necesite realizar un producto o contenido audiovisual para visibilizar, difundir y socializar alguna circunstancia histórica, biográfica, política o sociocultural determinada, las acciones específicas propias de una metodología vinculada a la Comunicología integrativa, tal como la planteamos, pueden ser las siguientes:

- El comunicólogo-gestor se involucra con una comunidad de interés para implementar un proyecto de intervención-acompañamiento dirigido a la resolución de un problema o necesidad comunicacional, ya sea porque sus servicios profesionales han sido requeridos o porque tiene un particular interés en dialogar con, e intervenir en

dicha comunidad.

- Una vez establecida la relación de colaboración con la comunidad en cuestión, se realiza un diagnóstico de la problemática a resolver, se revisa la pertinencia de los posibles métodos de intervención, se seleccionan las herramientas y tácticas mediáticas idóneas y se acuerdan con la comunidad las acciones, el proyecto a realizar.

- Se establece el tipo de intercambio a entablar entre la comunidad y el comunicólogo-gestor.

- Desde la primera sesión de trabajo, el comunicólogo-gestor solicita permiso y hace un registro audiovisual a manera de video diario o etnografía.

- Se consigue el equipo, los espacios, la infraestructura necesaria para la consecución de los objetivos de la comunidad de interés, organización o colectividad. Se sugiere el uso de recursos libres y de código abierto para la producción audiovisual.

- Se emprende un proceso de alfabetización mediática e informacional básica, enfocada en el tipo de audiovisual, de cinematografía o de producto mediático que sea pertinente para que la comunidad realice. 


\section{-}

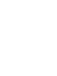

- Se congrega una unidad de producción audiovisual comunitaria (se le puede llamar UPAC, Tropa Audiovisual, o cualquier nombre que resulte sugerente a la colectividad), donde recae la responsabilidad de materializar las ideas generadas desde el diálogo colectivo.

- Se establecen los géneros y formatos más pertinentes; se realiza el o los productos audiovisuales.

- Se diseña una estrategia creativa de difusión, haciendo uso de plataformas digitales.

- Se evalúan los resultados, las ganancias o las pérdidas finales del proyecto, en términos de capital cultural, simbólico y económico.

- Al final del proyecto se puede tener: -El o los productos audiovisuales realizados por la comunidad.

-Una serie de proyecciones y presentaciones en espacios públicos o privados, o en medios masivos de información, en los que se involucre la comunidad y/o a públicos más amplios; o una página web, blog, cuentas en redes sociales o cualquier plataforma digital que permita difundir la película y el proyecto.

-Una etnografía audiovisual, un "detrás
José Luis Martin Galindo

de cámaras" que sirva como herramienta de análisis al investigador y como memoria del proceso para la propia comunidad.

-Elementos para una sistematización de la experiencia.

Es así que el modelo básico de la metodología que aquí se bosqueja funciona a partir de cuatro ejes de acción productiva, creativa, didáctica y estratégica, que será necesario poner en práctica desde una lógica flexible, fluida, adaptativa, nunca como recetas para prescripción alguna, que articulan una espiral constructiva: observación, representación, sistematización y articulación.

\section{A MANERA DE CIERRE}

Desde la Comunicología integrativa se piensa en la figura de un comunicólogo-gestor que acompaña proyectos de intervención social para ayudar a que comunidades de interés desarrollen su potencial comunicacional, a que organicen sus propias reglas del juego relacionadas con las puestas en común mediadas por los sistemas de información y los sistemas de comunicación, sobre los cuales se constituye, si no la sociedad en general, sí nuestras colectividades en particular, teniendo presente que "a través de la historia, la comunicación y 


\section{-}

.

la información, han sido las fuentes fundamentales de poder y contrapoder, de dominación y de cambio social, y esto es porque las batallas de poder se entablan en las mentes de las personas. La forma en que pensamos determina en último momento lo que hacemos" (Castells, 2010, 28:39-29:04).

Y es así que a partir de la revisión y reapropiación de ciertos elementos teóricos, técnicos y metodológicos de la Ciencia de la Comunicación contemporánea, así como la tradiciones de los medios ciudadanos herederos de la teoría crítica en América Latina, se pretende lograr una síntesis teórico-práctica que permita desarrollar una metodología dirigida hacia un diálogo constructivo de lo social entre producción académica-científica, producción creativa-mediática y pensamiento es- tratégico, para sintetizar un método de investigación-acción desde la especificidad comunicológica, que se viene visualizando bajo el concepto Comunicología integrativa.

Recurrir a la producción audiovisual como herramienta de investigación-intervención social implica desarrollar una praxis transversal que se articule desde la dimensión mediológica, es decir de difusión, pero que entre también en los ámbitos de la interacción y la expresión, para lograr resultados que se materialicen en la dimensión de la estructuración, mediante una especie de construccionismo comunicológico de lo social, en el que la producción audiovisual y mediática en general puede operar al servicio de la tríada cienciaarte-ingeniería social. 


\section{FUENTES CONSULTADAS}

Barranquero, A. (2014). En: Beltrán L. R. Comunicología de la liberación, desarrollismo y politicas públicas (introducción). Recuperado el 16 de julio de 2015 de http://bit.ly/2aWsVUQ.

Castells, M. (2010, noviembre 08). Presentación del libro Comunicación y poder a cargo de Manuel Castells [archivo de video]. Recuperado FALTA FECHA DE RECUPERACIÓN de http://bit.ly/2rpNzmZ.

Dagnino E. (2004). Sociedad civil, participación y ciudadanía en Brasil. Recuperado el 10 de agosto de 2015 de http:/ / bit.ly/2pXf8UM.

Galindo, J. (2005). "Sobre comunicología y comunicometodología. Primera guía de apuntes sobre horizontes de lo posible”. En: Culturales, I (1), 7-28.

- (2009). "Comunicología, comunicación y cultura. Exploración histórica de dos conceptos centrales en el tránsito del siglo XX al siglo XXI”. En: Razón y Palabra, núm. 66. Recuperado el 13 de junio de 2015 de http:/ / bit.ly/1arGUMf.

- (2011). Ingeniería en Comunicación Social y Deporte. Ciudad de México, Distrito Federal: Instituto de Altos Estudios sobre Deporte Cultura y Sociedad, AC (INDECUS, AC).

- (2014). Ingeniería en Comunicación Social, hacia un programa general. Puebla, México: Centro de Estudios en Comunicación Política-Benemérita Universidad de Puebla.

- e Islas, O. (coordinadores). (2015). Ingeniería en Comunicación Social y Comunicación Estratégica. Tenerife: Sociedad Latina de Comunicación Social.

. (2015). Presentación del número 90 de Razón y Palabra. El GICOM. Grupo de Ingeniería en Comunicación Social. Recuperado el 6 de julio de 2016 de http://bit.ly/2igvsMW.

Galindo Cáceres, J. (2017). El GICOM. "Grupo Ingeniería en Comunicación Social. Construyendo una experiencia de trabajo en colectivo”. En: Razón y Palabra, 21(1-96), 3-20. Recuperado el 29 de julio de 2017 de http:/ / bit.ly/2uNunyK.

Gumucio Dagron, A. (2011). "Comunicación para el cambio social: clave del desarrollo participativo". En: Signo y Pensamiento, 30(58), 26-39. Recuperado el 20 de julio de 2017 de http:/ / bit.ly/1JT0jAw.

- [TalachaVideo]. (2011b, marzo 04). Comunicación y cambio social-Alfonso Gumucio [archivo de video]. Recuperado el 01 de julio de 2017 de http://bit.ly/2q54XcC. 
Macías, N. y Cardona D. (2006). Comunicología como ciencia aplicada. Recuperado el 21 de julio de 2016 de http://bit.ly/2r4oDBI.

- (2011). “¿Formar comunicadores o comunicólogos?” En: Revista Mexicana de Comunicación, junio, n/a. Recuperado el 27 de junio de 2016 de http://bit.ly/114jf9y.

Mateos, C., y Gaona, C. (2015). "Constantes del videoactivismo en la producción audiovisual. Rastreo histórico (1917-2014) y puntualizaciones para una definición”. En: Sierra F. y Montero D., Videoactivismo y movimientos sociales. Teoría y praxis de las multitudes conectadas. 1st ed., 106-140. Barcelona: Editorial Gedisa.

Morales, S. (2009). "La apropiación de TIC: una perspectiva". En: Los jóvenes y las TIC. Córdoba: UNC. - (2011). "Acceso y apropiación: una apuesta de política pública". En: Enseñar con Tecnologias. Nuevas miradas en la formación docente. Buenos Aires: Prometeo.

Rizo, M. (2007). "Interacción y comunicación en entornos educativos: Reflexiones teóricas, conceptuales y metodológicas". En: E-Compos abril, 1-16. Recuperado el 30 de julio de 2017 de http://bit.ly/2bXXFCx

Rodríguez, C. (2009). "De medios alternativos a medios ciudadanos: trayectoria teórica de un término". Folios Revista de la Facultad de Comunicaciones de la Universidad de Antioquia, núm. 21 y 22, 13-25.

Téllez, P. [Periodismo de hoy]. (2013, noviembre 15). Comunicación, ciudadanía y medios comunitarios / Patricia Téllez, [archivo de video]. Recuperado FALTA FECHA DE RECUPERACIÓN de http://bit.ly/2q7Eydq.

Torrico, E. (2010). Luis Ramiro Beltrán: por una Comunicología de liberación. Recuperado el 17 de julio de 2016 de http://bit.ly/2q7INFU. 